Reprod. Nutr. Dévelop., 1983, 23 (1), 25-39.

\title{
Etude des glucides totaux et des glycoprotéines endogènes des contenus intestinaux chez le veau préruminant
}

\author{
J. M. BESLE, M. POUGHEON, E. BAYLE, P. THIVEND \\ Laboratoire de la Digestion des Ruminants, I.N.R.A., \\ Theix, 63110 Beaumont, France.
}

\begin{abstract}
Summary. A study of total endogenous carbohydrates and glycoproteins in the intestinal contents of the preruminant calf.
\end{abstract}

The aim of this work was to determine neutral and conjugated endogenous carbohydrates in different parts of the intestine of the preruminant calf. Two animals, fitted with ileal and colonic cannulae, were fed whole milk containing chromic oxide on a cellulosic support as a marker. Samples were collected from ileum, colon and faeces when the calves were 1 and 2 months old. Carbohydrates were determined (fig. 1) using colorimetric determination and ion-exchange (IEC) or molecular sieve (MCS) chromatography. The water-soluble and the insoluble carbohydrate fractions were chromatographically determined after hydrolysis and corrected to include polymers. Glucose polymers were not included in the insoluble fraction because they could not be separated from the glucose resulting from the cellulosic support of the marker. A sensitive automatic method was developed to determine proteins (see Appendix and figure 5).

Lactose was completely hydrolyzed at the end of the small intestine. Total carbohydrates (table 1) accounted for $20 \%$ of dry matter (DM), half of which was watersoluble in the ileum, and decreased in the faeces $110 \% \mathrm{DM}, 90 \%$ of which was insoluble). In the ileum, the water-soluble fraction (table 1) contained a few monosaccharides (1 \% DM) and mainly oligo and polysaccharides (free and unconjugated) composed of galactose, glucose, mannose, fucose and ribose $(62,16,7,5,1 \%$ total sugars, respectively). The insoluble fraction had the same composition $(63$, undetermined, 19 , traces, $3 \%$ total sugars, respectively). The same components were found in the large intestine but in different amounts.

In the ileum of a 1-month-old calf, the water-soluble fraction isolated by MSC (fig. 2, table 2) contained the following :

1) $\mathrm{di}$, tri and tetra-saccharides (38\% of total water-soluble sugars or $0.7 \%$ of lactose intake) that were isolated and studied separately (fig. 3). They were found to be both neutral and reducing sugars composed of glucose and galactose in proportions which led to the formula proposed in table 3 ;

2) 11 glycoproteins and peptides (15\% DM) whose main fraction (peak $X$ ) amounted to $47 \%$ of the total water-soluble sugars ; $80 \%$ of these sugars were conjugated. In the colon and faeces, the same components were found (but in different proportions) along with other oligosaccharides in lesser amounts.

As the age of the calves increased, the water-soluble carbohydrate content of all the digesta decreased. The proportion of the $X$ fraction increased at the expense of the oligosaccharides, whereas insoluble glycans varied slightly. The O-glycoproteins (mucoproteins) were progressively replaced by $\mathrm{N}$-glycoproteins (rich in mannose) from digestive tract cell wall fragments and microorganisms. This was indicated by a decrease 
in galactose-fucose and galactose-mannose ratios in glycoproteins and by an increase in insoluble ribose in the large intestine. Oligosaccharides came from products of lactose hydrolysis and were built up by lactase which acted as a synthetase (as noted by Roberts and Mc Farren, 1953).

\section{Introduction.}

Les glucides et les glycoprotéines d'origine endogène présents dans la lumière intestinale chez le veau préruminant sont mal connus. Leur étude s'avère cependant utile, d'une part à cause de leurs fonctions physiologiques, d'autre part pour mieux préciser la digestion, dans les différentes parties de l'intestin, des glucides complexes des aliments d'allaitement. Gaillard et Van Weerden (1976), Besle, Lassalas et Thivend (1980) ont observé une digestibilité négative des mannanes et galactanes hydrosolubles de la levure d'alcanes à la fin de l'intestin grêle. Ce phénomène peut-il s'expliquer par la présence de glucides endogènes?

Les glucides du lait entier sont constitués principalement de lactose qui est entièrement digéré dans l'intestin grêle ainsi que de polyosides libres et conjugués (respectivement 1 et $0,26 \mathrm{~g} / \mathrm{l}$ selon Montreuil et Kobus, 1960). Ces fractions, quantitativement peu importantes, sont sans doute dégradées par la microflore avant la fin de l'iléon. Nous pouvons donc les négliger dans une première approche et discuter de l'incidence de leurs produits de dégradation sur nos résultats. Dans ces conditions la fraction glucidique du contenu intestinal de l'animal nourri au lait peut fournir une estimation satisfaisante des glucides et glycoprotéines endogènes. Ils auraient pour origine, sur le plan physiologique, les sécrétions épithéliales (mucoprotéines, immunoglobulines...), les desquamations et la microflore. Dans le contenu intestinal on trouve un mélange de ces composés sous forme intacte, dégradée ou transformée. Le but de ce travail a été de déterminer, qualitativement et quantitativement, l'importance de cette fraction à la fin de l'intestin grêle et dans le gros intestin, en utilisant des animaux fistulés. Le résumé de ces résultats a été publié antérieurement (Besle et al., 1981).

\section{Matériel et méthodes.}

Animaux, régimes, prélèvements.

Deux veaux de race Frisonne ont été munis à l'âge de 12 jours d'une canule double réentrante, iléo-iléale, et d'une canule simple dans le côlon proximal. Ils ont reçu exclusivement du lait de vache contenant comme marqueur de transit de l'oxyde de chrome $(0,25$ à 0,50 p. 100 de la matière sèche) sur support de poudre de cellulose (100 g p. $30 \mathrm{~g}$ d'oxyde de chrome). L'aliment était distribué au seau à raison de deux repas par jour, les quantités distribuées augmentant à chaque période en fonction du poids métabolique (48 $\mathrm{g}$ de matière sèche $/ \mathrm{kg} \mathrm{p}^{0,75}$ ) des animaux.

Aux âges de 1 et 2 mois (poids moyens respectifs de 53 et $75 \mathrm{~kg}$ ), des échantillons de contenu digestif et de fèces ont été collectés pendant trois jours 
consécutifs, à intervalle de $6 \mathrm{~h}$ avec un décalage quotidien de $2 \mathrm{~h}$ (soit un prélèvement toutes les 2 heures pendant $24 \mathrm{~h}$ ), immédiatement congelés et réunis en échantillons moyens par niveau du tube digestif. Ils ont ensuite été lyophilisés et broyés.

\section{Méthodes d'analyse.}

Les méthodes de dosage de l'oxyde de chrome et de détermination des glucides (extraction aqueuse, dosage des sucres totaux et réducteurs, chromatographie par tamisage moléculaire et par échange d'ions) sont décrites dans deux mémoires antérieurs (Besle, 1974 ; Besle, Lassalas et Thivend, 1981). Les conditions de l'extraction aqueuse (inactivation des enzymes par l'éthanol $95^{\circ} \mathrm{GL}$ au bain-marie bouillant puis extraction aqueuse à $40^{\circ} \mathrm{C}$ ) sont justifiées dans cette étude car, selon Snary et Allen (1972), ni I'action de l'alcool, ni celle de la chaleur $\left(100^{\circ} \mathrm{C}\right)$ ne modifient les propriétés physicochimiques des mucoprotéines. $L^{\prime}$ isolement de la fraction neutre se fait après passage sur résines par la méthode de Montreuil (1957) modifiée par Besle et Pitiot (1976). Les groupements protéiques hydrosolubles ont été dosés par la méthode automatisée décrite en annexe.

Le fractionnement glucidique est décrit dans la figure 1. L'effluent de colonne par tamisage moléculaire (CTM) a été divisé pour le dosage simultané des sucres totaux, des sucres réducteurs et des protéines.

L'étude complète par chromatographie d'échange d'ions (CEI) de la composition glucidique des contenus digestifs et des fèces a été effectuée sur les deux

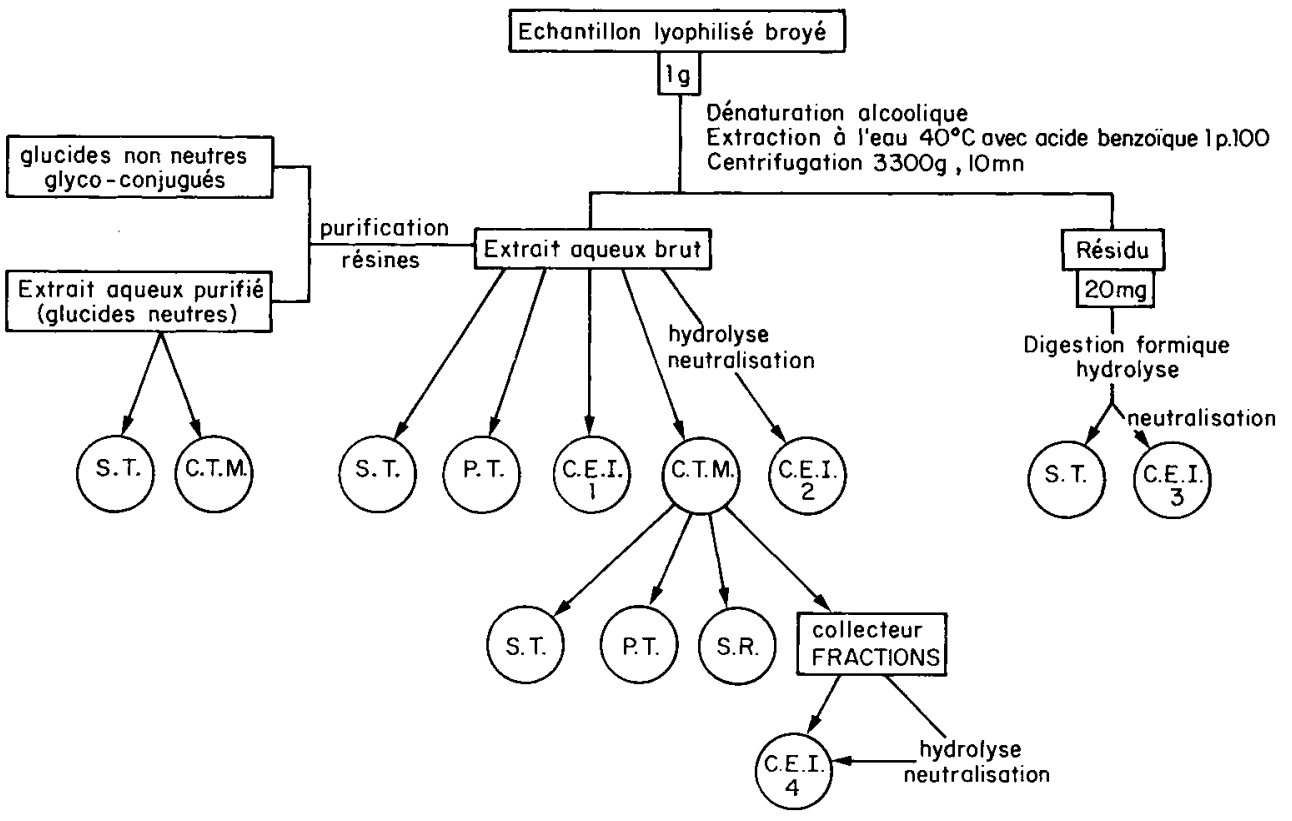

FIG. 1. - Schéma du fractionnement glucidique.

ST = dosage global des sucres totaux par rapport au glucose; $\mathrm{SR}=$ sucres réducteurs; PT = dosage global des Protéines Totales par rapport à la sérum albumine bovine ; $\mathrm{CTM}=$ Chromatographie par Tamisage Moléculaire ; CEI = Chromatographie par Echange d'lons. 
veaux. L'étude par tamisage moléculaire a été réalisée sur tous les échantillons provenant du veau $n^{\circ} 1$. Sur l'autre animal nous avons limité les analyses CTM à quelques échantillons (iléon, côlon, fèces) qui ont donné, qualitativement et quantitativement, des valeurs identiques à celles obtenues avec le premier animal. C'est la raison pour laquelle les résultats correspondant à l'analyse par tamisage moléculaire ont été donnés sur un seul animal.

L'hydrolyse des extraits aqueux, comme celle des fractions collectées, a été réalisée par $\mathrm{H}_{2} \mathrm{SO}_{4} 3 \mathrm{~N}$ pendant $4 \mathrm{~h}$ à $120^{\circ} \mathrm{C}$. Dans ces conditions, établies après une cinétique d'hydrolyses : 1) la libération des oses est optimale ; 2) les taux de dégradation du galactose, du glucose, du mannose, du fucose et du ribose sont respectivement de $0,6,0,5$ et 25 p. $100 ; 3$ ) les liaisons osaminidyl sont coupées, les radicaux acétyl sont libérés; les acides sialiques sont détruits (Montreuil et Spik, 1968) ; 4) le Brij contenu dans l'effluent CTM n'interfère pas. Après neutralisation au borate de sodium et à la soude, ces hydrolysats ont été chromatographiés par $\mathrm{CEl}$.

Les résidus d'extraction aqueuse (glucides insolubles) ont été hydrolysés (digestion à l'acide formique et hydrolyse sulfurique) et neutralisés par la méthode décrite par Besle, Lassalas et Thivend (1980). Dans ces conditions, les liaisons osaminidyl sont coupées, les acides sialiques détruits (Montreuil et Spik, 1968), le ribose est seul, parmi les oses déterminés, à être dégradé à un taux de 9 p. 100. Les glucides des hydrolysats sont ensuite dosés globalement et chromatographiés par CEI suivant les méthodes décrites précédemment.

\section{Résultats.}

1) Teneur des digesta en glucides et composition des hydrolysats (tabl. 1).

La teneur en glucides insolubles (dosage global des sucres totaux ST) évolue peu dans l'intestin (en moyenne 9 p. 100 de la matière sèche). La partie hydrosoluble (ST) diminue de l'iléon aux fèces (en moyenne respectivement de 53 à 9 p. 100 des glucides totaux). Elle contient une fraction glucidique neutre qui est relativement plus importante dans l'iléon que dans les fèces (en moyenne respectivement 67 et 28 p. 100 des glucides hydrosolubles totaux).

Les polyosides hydrosolubles (neutres et liés à d'autres molécules) constituent en moyenne environ 9 p. 100 de la matière sèche (MS) dans l'iléon, et 10 fois moins dans les fèces (tabl. 1). Ils donnent, après hydrolyse lanalyse chromatographique CEI 2 ; fig. 1 et tabl. 1) principalement du galactose et du glucose, mais aussi du mannose et du ribose et des sucres inconnus. Les proportions du glucose et du ribose augmentent dans le gros intestin, tandis que celles du galactose diminuent.

Les polyosides insolubles (glucose mis à part) constituent en moyenne environ 3 p. $100 \mathrm{MS}$ dans l'iléon et ne diminuent guère dans le gros intestin (1,6 p. $100 \mathrm{MS}$ ). Ils sont composés (CEI 3 ; fig. 1 et tabl. 1) principalement de galactose, de mannose et de ribose. Le long de l'intestin, les proportions de mannose et de ribose augmentent au détriment du galactose. Le rapport molaire $\mathrm{gal} /$ man diminue en moyenne à l'âge d'un mois, de 3,5 à 2,1. 


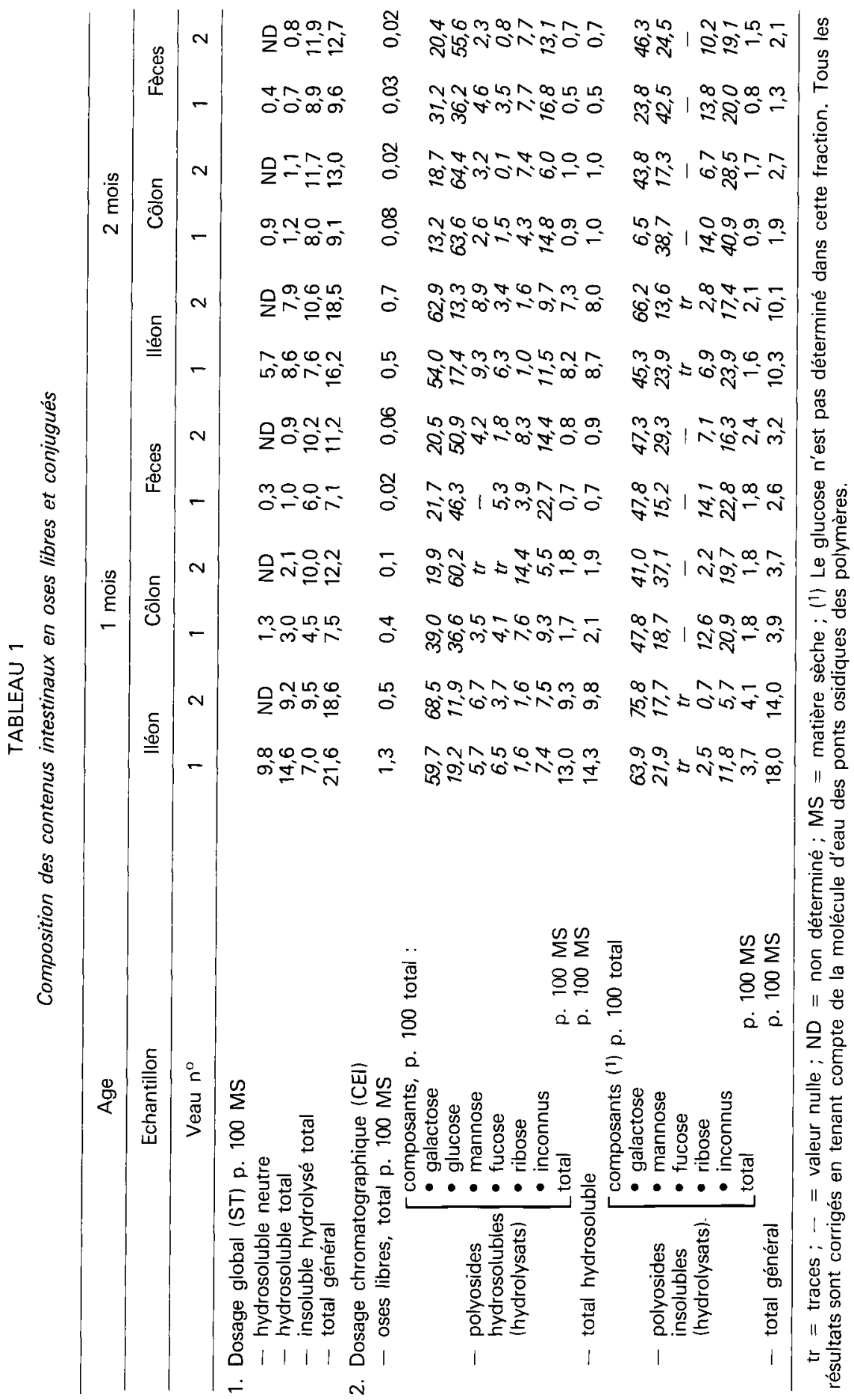


La différence entre le dosage global et celui obtenu après chromatographie, faible dans la fraction hydrosoluble, est importante dans le résidu insoluble. Elle provient sans doute de composés glucidiques réagissant par dosage des sucres totaux mais non évalués par CEI : acides uroniques, osamines, polyosides solubilisés mais incomplètement hydrolysés...

2) Nature des glucides et des glycoprotéines hydrosolubles.

a) Lactose et osés libres.

Dans les contenus iléaux (tabl. 1), nous ne trouvons que des traces de lactose et très peu d'oses libres (au maximum 1,3 p. $100 \mathrm{MS}$ ), constitués principalement de galactose et de glucose (en moyenne 59 et 26 p. 100 des oses), avec des quantités variables de mannose, fucose, ribose. Leur concentration diminue encore dans le gros intestin, le glucose et le ribose devenant prépondérants.

b) Oligosides.

- Dans l'iléon nous avons séparé les di, tri et tétraosides B1, C1, D1, réducteurs, représentant 38 p. 100 des glucides hydrosolubles et 0,7 p. 100 du lactose ingéré (fig. 2 ; tabl. 2). Si on trace la courbe log Poids moléculaire (PM)
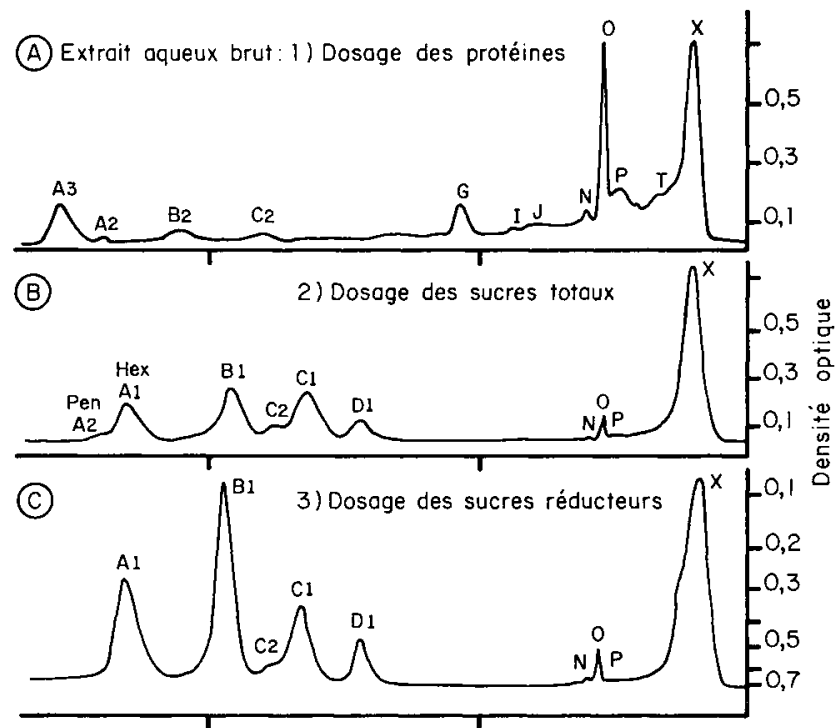

(D) Extrait aqueux neutre: Dosage des sucres totoux

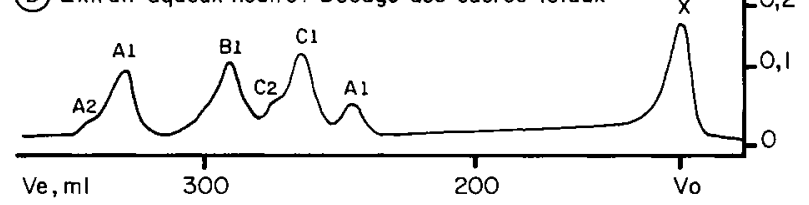

FIG. 2. - Exemple de séparation par tamisage molécuiaire sur biogel d'un extrait aqueux brut et neutre de contenu d'iléon (veau $n^{0} 1,2$ mois).

$\mathrm{Hex}=$ hexoses, Pen = pentoses ; A, B, C ...X $=$ fractions correspondant aux DP (oligosides) $1,2,3 \ldots>22 ; \mathrm{Ve}=$ volume d'éluant, $V_{0}=$ volume mort, débit $=1,12 \mathrm{ml} / \mathrm{min}$; Chromatogrammes : $\mathrm{A}$ et $\mathrm{B}=$ échelle linéaire, $\mathrm{C}=$ échelle semi-logarithmique inversée, $\mathrm{D}$ : échelle semilogarithmique. 


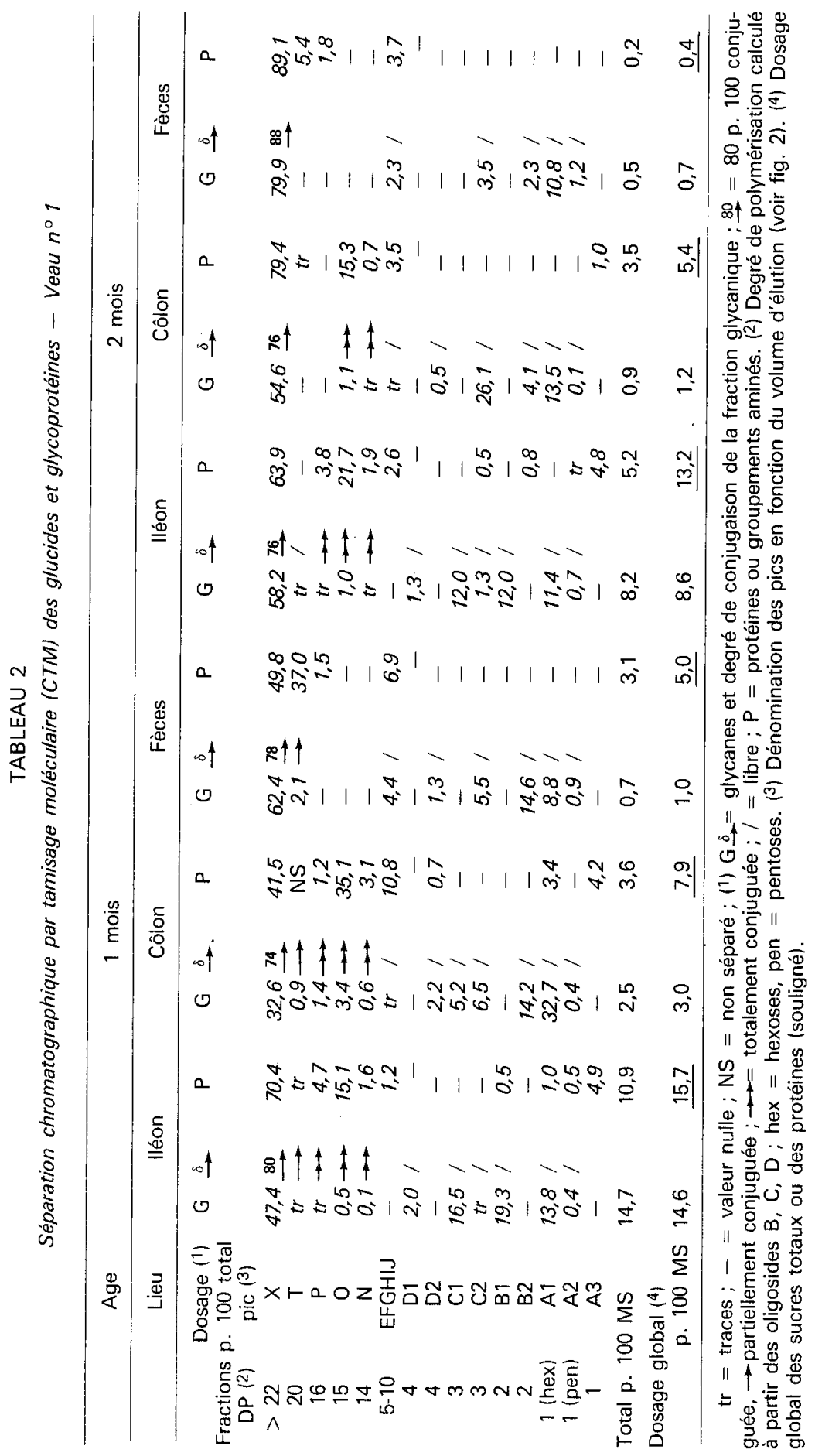

Reproduction, nutrition, développement, $n^{\circ} 1$ 
$=\mathrm{F}$ (volume d'élution/volume mort), on constate que ces 3 pics sont alignés sur une droite différente de celle des dextrines et proche de celle des $\alpha$-galactosides (raffinose, stachyose, verbascose, ...). La fraction B1, isolée, se décompose par $\mathrm{CEI}$, en traces de lactose et 6 autres pics dont 5 importants (fig. 3). Son hydrolyse donne en totalité du galactose et du glucose dans un rapport molaire $5 / 1$. Cette répartition, ainsi que d'autres considérations qui seront discutées plus loin, nous autorisent à proposer les formules indiquées au tableau 3. De la même manière, les fractions $\mathrm{C} 1$ et D1 se décomposent ainsi que l'indique la figure 3. Par hydrolyse, elles donnent des traces de lactose ainsi que du galactose et du glucose dans les rapports molaires respectifs de $2 / 1$ et $2 / 2$, ce qui nous permet de proposer les formules indiquées au tableau 3.

- Dans le gros intestin, les oligosides diminuent quantitativement (21 p. 100 des glucides hydrosolubles dans les fèces à l'âge d'un mois) et sont constitués, en plus de ceux décrits précédemment, de di, tri, tétraosides $B 2, C 2$, D2 ainsi que d'oligosides $E, F, G, H$, J présents, en faible quantité, surtout dans les fèces (tabl. 2). Les glucides B, C, D représentent respectivement 0,1 et 0,01 p. $100 \mathrm{du}$ lactose ingéré dans le côlon et les fèces. Seules les fractions B2 et C2 ont été isolées et étudiées (tabl. 3), les autres étant présentes en trop faibles quantités.

TABLEAU 3

Composition de quelques fractions glycaniques (1) - Veau $n^{\circ} 1$

Oligosides réducteurs neutres

\begin{tabular}{lccccc}
\hline Fraction & $\mathrm{B}_{1}$ & $\mathrm{~B}_{2}$ & $\mathrm{C}_{1}$ & $\mathrm{C}_{2}$ & $\mathrm{D}_{1}$ \\
\hline Origine-âge $\left(^{2}\right)$ & iléon-1 & côlon-2 & iléon-1 & côlon-1 & iléon-1 \\
Rapport gal/glc & $5 / 1$ & $1 / 1$ & $2 / 1$ & $0 / 3$ & $2 / 2$ \\
$\begin{array}{l}\text { Nombre de pics } \\
\text { par CEl (3) }\end{array}$ & 5 & $\mathrm{ND}$ & 2 & $\mathrm{ND}$ & 1 \\
$\begin{array}{l}\text { Formule possible } \\
\text { tr lactose }\end{array}$ & & & & \\
& gal-gal & gal,glc & gal-gal-glc & glc-glc-glc & gal,glc,gal,glc \\
& glc-glc & & & & \\
\hline
\end{tabular}

Fraction hydrosoluble $X$ (âge d'1 mois)

\begin{tabular}{lcccccc}
\hline & \multicolumn{2}{c}{ Origine : } & \multicolumn{3}{c}{ Gros intestin } \\
\cline { 2 - 6 } & p. 100 molaire & p. 100 OP ${ }^{(4)}$ & p. 100 molaire & p. 100 OP (4) \\
\hline galactose & 65,8 & 57 & 64,3 & 60 \\
glucose & 11,2 & 30 & 20,0 & 20 \\
mannose & 9,4 & 85 & 3,5 & 35 \\
fucose & 13,6 & 99 & 10,1 & 81 \\
ribose & - & & - & 2,0 & \\
gal/fuc & & 4,8 & & & 6,4 & \\
gal/man & & 7,0 & & & 19,0 & \\
\hline
\end{tabular}

$\operatorname{tr}=$ traces $;-=$ valeur nulle $; N D=$ non déterminé $;(1)$ gal = galactose, glc $=$ glucose, fuc $=$ fucose, man $=$ mannose $;(2)$ Contenu digestif et âge de l'animal en mois ; $\left.{ }^{3}\right) \mathrm{CE}=\mathrm{chroma-}$ tographie par échange d'ions ; (4) p. 100 de l'ose sous forme polymérisée dans l'extrait aqueux. 
Les pics 1, 2, 3 qui apparaissent sur les chromatogrammes CEI (fig. 3) sont dus probablement à des impuretés de l'effluent CTM (Brij, molécules organiques du Biogel...), mais il ne faut pas exclure la présence de composés glycosaminés, produits de dégradation des fractions glycaniques, bien que ceux-ci réagissent faiblement à l'orcinol sulfurique.

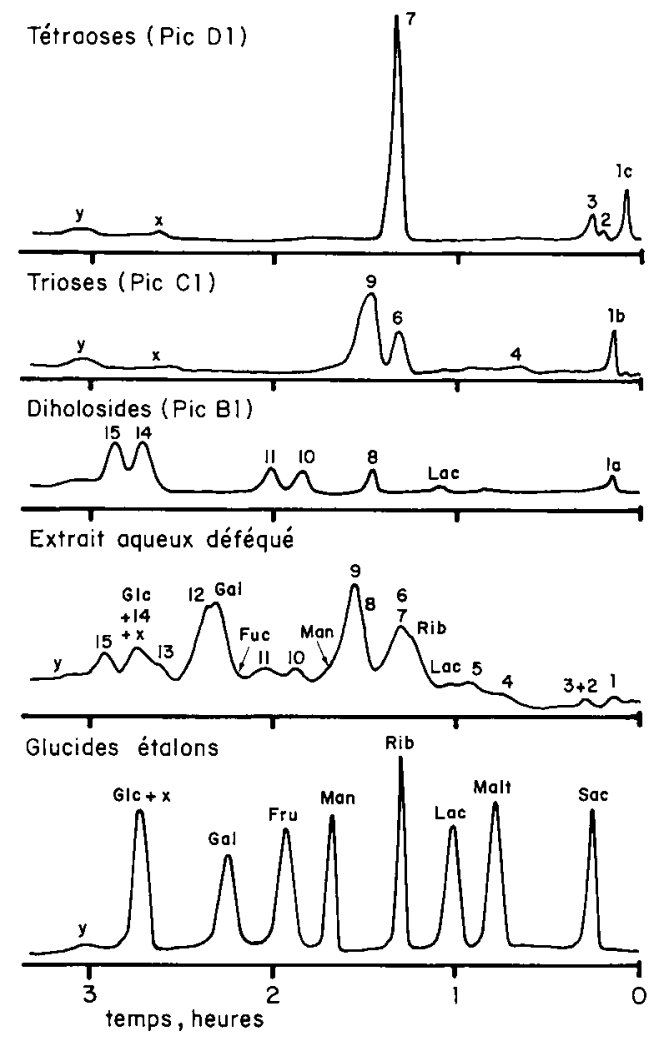

FIG. 3. - Analyse par chromatographie échangeuse d'ions (CEI) d'un extrait aqueux de contenu iléal déféqué (veau $n^{\circ} 1$ à 2 mois), et de quelques fractions collectées (pics B1, C1, D1) en sortie de colonne Biogel (CTM).

$x, y=$ artéfacts provenant du gradient d'élution; 1a, 1b, 1c, 2, 3... $15=$ glucides inconnus. Temps, heures $=$ temps net de l'élution. Conditions opératoires : Résine Technicon (S), gradient D modifié (Besle, 1974), débit $=1 \mathrm{ml} / \mathrm{min}$. Les glucides de l'extrait aqueux (déféqué) ont été identifiés par leur temps de rétention, par surcharges et par d'autres gradients d'élution, avec les résines Technicon (S) et Biorad (Aminex A15), qui ont permis de séparer le ribose et le mannose des pics inconnus, ainsi que le fucose du galactose.

\section{c) Peptides et glycoprotéines.}

Le dosage de l'effluent au réactif de Lowry en sortie de colonne CTM fait d'abord apparaître des petites molécules (acides aminés, peptides...) indépendantes des glucides aux niveaux A3, A2, B, C, D, G (fig. 2 ; tabl. 2) qui disparaissent pratiquement dans le gros intestin. Nous observons ensuite 3 autres pics : 
$\mathrm{N}$, O (le plus important) et $\mathrm{P}$, correspondant aux degrés de polymérisation (DP) d'environ 14-16, réducteurs, réagissant aussi au dosage des sucres totaux, et qui disparaissent après purification (fig. 2, A et $D$ ) ; ce sont donc vraisemblablement des glycopeptides. Enfin deux pics, mal séparés $T$ et $X$, sont élués en exclusion et ont les mêmes caractéristiques que les 3 précédents. II s'agit sans doute d'un mélange de glycanes neutres et de glycoprotéines, puisqu'après purification il reste encore environ 22 p. 100 des glycanes de $X$.

La composition osidique de la fraction $X$ (tabl. 3) montre qu'elle contient la quasi-totalité du fucose. Dans le gros intestin, les teneurs en glucose et en ribose croissent ; les rapports molaires entre galactose (gal)-fucose (fuc), et galactosemannose (man), augmentent respectivement de 4,8 à 6,4 et de 7,0 à 19,0. Cette fraction représente une part croissante des glycanes hydrosolubles, pour une proportion pratiquement inchangée de glycoconjugués. Notons à titre de comparaison que la fraction gal/man est plus faible dans le résidu insoluble et varie peu entre le contenu iléal et les fèces (de 3,0 à 2,8 ).

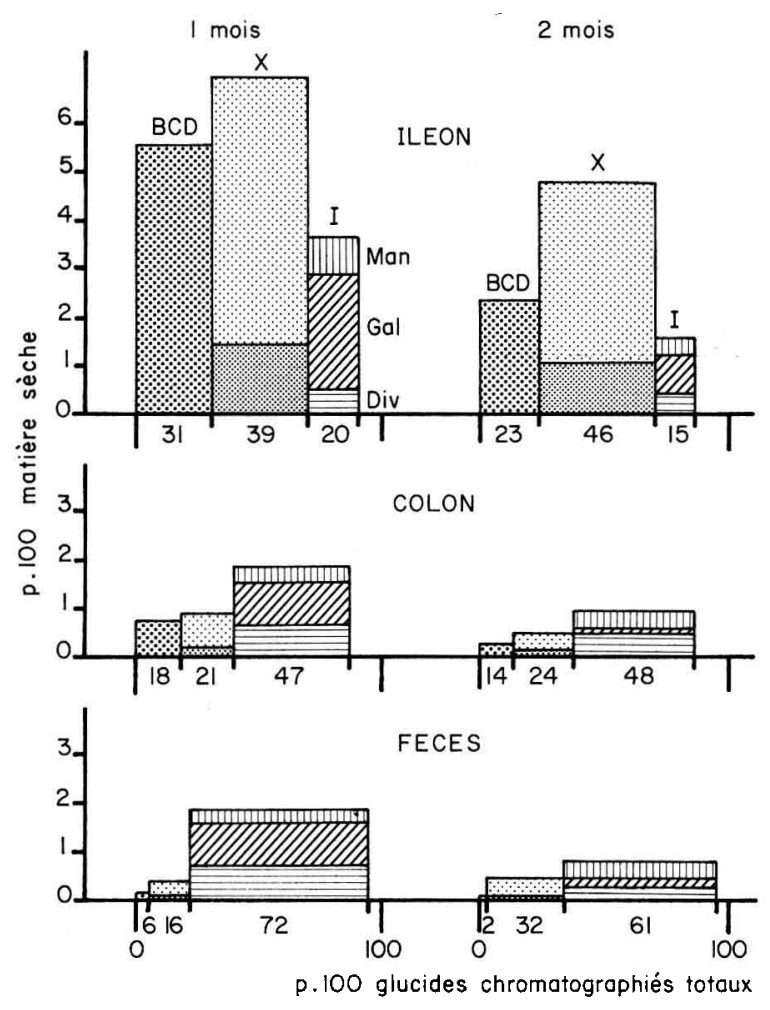

$$
\begin{gathered}
\text { Fraction hydrosoluble }\left\{\begin{array}{l}
\text { oligosides } \mathrm{BCD} \\
\text { glycanes } X \text { conjugues } \\
\text { glycanes } X \text { neutres }
\end{array}\right. \\
\text { Fraction insoluble (I) }\left\{\begin{array}{l}
\text { mannose } \\
\text { galactose } \\
\text { divers }
\end{array}\right.
\end{gathered}
$$

FIG. 4. - Variation de la composition glucidique des contenus intestinaux du veau $n^{\circ} 1$ en fonction du site et de l'âge. 
Nous observons enfin que la concentration en protéines totales hydrosolubles diminue le long de l'intestin, parallèlement à celle des glucides. La différence entre le dosage global des protéines et l'évaluation chromatographique est sans doute due au fait que nous n'avons pu quantifier la " traînée de pic " en exclusion qui se prolonge jusqu'au pic $N$. Les différences plus faibles observées pour la fraction glycanique sont sans doute principalement dues au mode d'expression (sucres totaux dosés à l'orcinol sulfurique par rapport au maltose) qui est forcément global car nous n'avons pas déterminé la composition de ces oses de tous les pics. Cependant l'erreur est vraisemblablement faible puisque les réactivités des principaux constituants (galactose, glucose, mannose) sont proches les unes des autres.

\section{3) Evolution avec l'âge (fig. 4 ; tabl. 1).}

Dans l'iléon, comme dans le gros intestin, nous observons une diminution des concentrations de glucides entre les âges de 1 et 2 mois, tant dans la fraction soluble que dans l'insoluble. La fraction soluble contient relativement plus de polymère $X$, celui-ci étant toujours à 80 p. 100 sous forme glycoconjuguée. Les constituants glucidiques suivent cette évolution, en particulier le mannose, dont la proportion augmente comme celle de $X$, tandis que celle du galactose diminue. Dans la fraction insoluble, la proportion de mannose suit une évolution inverse chez les deux animaux, celle du ribose augmente au détriment de celle du galactose. Enfin, nous observons à la fin de l'intestin grêle et dans le gros intestin une diminution de la concentration en protéines solubles.

La diminution avec l'âge des composés étudiés peut s'expliquer en partie par l'accroissement de leur dégradation enzymatique (Ternouth, Siddons et Toothill, 1971 ) et microbienne, et en partie, sans doute, par la diminution de leur production. En effet, chez l'agneau, entre 1 et 5 semaines d'âge, Moon et Joel (1975) et Attaix (1979) ont observé une diminution, dans le tissu intestinal, respectivement de la trophicité des cellules et de la synthèse protéique.

\section{Discussion.}

Origine des oligosides et incidence sur la digestion du lactose. - Une partie du galactose et du glucose n'est pas absorbée directement après hydrolyse du lactose par la lactase et est présente dans la lumière intestinale (Coombe et Smith, 1973). Il est possible que ces oses soient alors utilisés par cette enzyme pour former les oligosides B, C, D et peut-être des homologues supérieurs. Cette hypothèse est justifiée par les travaux de Aronson (1952) (in vitro) et de Roberts et Mc Farren (1953) (in vitro et in vivo sur rats), ces derniers ayant isolé des oligosides du même type. D'après Wallenfels (1951), la lactase agirait comme une galactosyl-transférase, ce qui justifierait les formules proposées dans le tableau 3. Le tétraoside $\mathrm{D}$ pourrait provenir de la condensation de 2 molécules de lactose sur le galactose terminal de l'une d'elles. Ces oligosides diminueraient légèrement (de 0,7 point) la digestibilité iléale du lactose. Ils contribuent à l'établissement de la microflore et pourraient peut-être expliquer les phénomènes de diarrhée observés chez l'agneau avec des régimes très riches en lactose (Peyraud, 1981). II est 
possible qu'une partie des diholosides $B$ (neutres et réducteurs) provienne de la dégradation des glucides complexes du lait : $\mathrm{N}$ acétyl lactosamine provenant des oligosides, galactose- $\mathrm{N}$ acétyl galactosamine faisant partie de la séquence des glycopeptides de la caséine kappa (Fournet et al., 1975).

Incidence sur la digestion des glucides complexes. - Le galactose, le glucose et le mannose conjugués de la fraction hydrosoluble qui constituent, en moyenne à l'âge d'un mois, respectivement $7,1,1,8$ et 0,7 p. $100 \mathrm{MS}$ dans l'iléon, doivent être pris en compte dans l'étude de la digestion des glucides complexes. Cette étude éclaire en particulier les résultats obtenus précédemment dans le cas d'un aliment d'allaitement contenant 35 p. 100 MS de levures d'alcanes (Besle, Lassalas et Thivend, 1980). Si I'on exprime la teneur des glucides intestinaux par rapport au lactose ingéré, cela explique la digestibilité iléale négative des galactanes et glucanes de la levure, et, justifie l'hypothèse selon laquelle les mannanes insolubles de la paroi seraient déjà partiellement solubilisés dans l'intestin grêle, étant donné la faible teneur en mannose endogène.

Evolution des glycanes complexes et des glycoconjugués. - Dans les contenus iléaux, la part des immunoglobulines serait très faible puisqu'elle ne représente que 0,1 p. 100 de la MS dans le jéjunum (Porter et Noakes, 1970). Par contre, si les glycopeptides du lait (provenant principalement de la caséine kappa selon Jenness, 1974) n'étaient pas dégradés dans l'intestin grêle, ils fourniraient environ un dixième du galactose des polyosides hydrosolubles, ainsi que de la $\mathrm{N}$ acétyl galactosamine et de l'acide $\mathrm{N}$ acétyl neuraminique (Fournet et al., 1975) qui ne sont pas dosés dans notre étude. Nous ne pouvons pas apprécier la fraction digérée des glycanes de la caséine kappa, aussi convient-il, par prudence, de minimiser la part du galactose dans les polymères. La fraction glycanique endogène hydrosoluble proviendrait surtout de mucoprotéines (Spiro, 1973). Les rapports molaires gal/fuc et gal/man de la fraction $X$ sont plus élevés que ceux trouvés dans d'autres extraits muco-protéiques (muqueuse gastrique d'agneaux : Huguet, Solère et Rémy-Heintz, 1975 ; de porc : Pallavicini et al., 1972 ; Snary et Allen, 1971 ; intestin grêle du porc: Degand, Gaveriaux et Havez, 1972). L'importance de ces rapports ainsi que leur accroissement dans le gros intestin auraient pour cause d'une part l'augmentation apparente de la proportion en O-glycoprotéines (pauvres en fucose et en mannose) et d'autre part la dégradation des fractions glycaniques (qui débuterait dans l'intestin grêle), qui ont le mannose et le fucose en position externe (Strecker et Montreuil, 1979 ; Bella et Kim, 1972). Globalement, l'augmentation de la fraction insoluble, qui devient prépondérante dans le gros intestin et s'enrichit en mannose ainsi qu'en ribose, montre l'importance croissante des desquamations et des micro-organismes. En effet les parois des cellules épithéliales de la muqueuse intestinale contiennent principalement des $\mathrm{N}$-glycoprotéines, riches en mannose (Parry, 1978), mais on y trouve aussi des O-glycoprotéines (Kathan et Adamanly, 1967) et des glycolipides (Falk et al., 1979). Le ribose, en majeure partie d'origine nucléique, est témoin de la richesse en cellules épithéliales et microbiennes. Nous savons en outre que les parois bactériennes contiennent peu de fucose (Jouany et Thivend, 1972), que la teneur en osamines (surtout en galactosamine) " endogènes " diminue dans le 
gros intestin du veau (Combe et al., 1980) et enfin que la flore microbienne croît dans ce compartiment digestif (Contrepois et Gouet, 1973).

En conclusion, nous avons montré que la quantité de glucides endogènes présents à la fin de l'intestin grêle est importante (environ 19 p. 100 de la matière sèche) ; elle diminue de moitié dans le gros intestin, la fraction insoluble devenant prépondérante. Elle est constituée d'oligosides synthétisés probablement à partir du lactose et de glycoconjugués. La teneur de ces composés diminue avec l'âge, entre 1 et 2 mois ; leur importance demande à ce qu'on les prenne en compte dans toute étude sur la digestion des glucides non laitiers incorporés dans les aliments d'allaitement utilisés par le préruminant.

Reçu en février 1982.

Accepté en août 1982.

Remerciements. - A Marie-Thérèse Beaufort, Michel Fabre, Jacques Lefaivre, Louis L'Hotelier, pour leur contribution à la réalisation de ce travail.

\section{ANNEXE}

\section{DOSAGE DES PROTÉINES SOLUBLES TOTALES}

Nous avons tout d'abord utilisé la méthode de Lowry et al. (1951) automatisée par Talmant (1970) pour doser en continu les protéines solubles totales, mais elle convenait mal pour deux raisons. La première était que le sulfate de cuivre en milieu tartrate double de $\mathrm{Na}$ et $\mathrm{K}$ se déposait dans les bobines de mélange et les encrassait, nécessitant des lavages périodiques (toutes les deux heures), difficiles à réaliser dans le dosage continu de l'effluent de CTM. La deuxième raison était que la solution de Brij 35 contenue dans l'éluat interférait et produisait une floculation. Nous avons donc modifié la méthode manuelle de Wang et Smith (1975), et I'avons automatisée. Avec le montage décrit dans la figure 5 , les inconvénients précités sont supprimés, la gamme étalon de sérum albumine bovine est linéaire de 50 à $700 \mu \mathrm{g} / \mathrm{ml}$ mais la sensibilité est deux fois moindre. L'emploi de

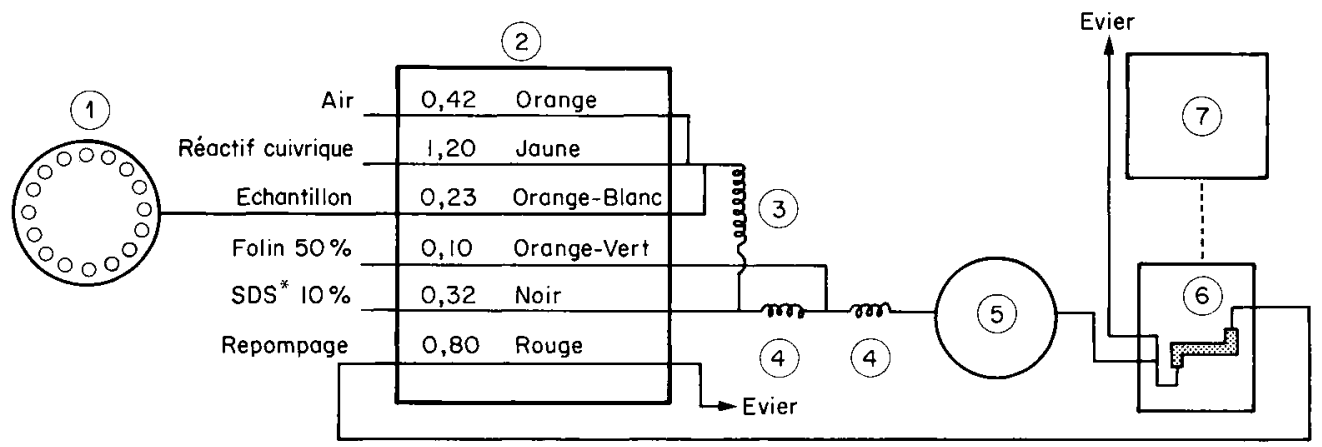

FIG. 5. - Circuit de dosage en continu des protéines hydrosolubles totales.

(1) Echantillonneuse. (2) Pompe péristaltique, débit $(\mathrm{ml} / \mathrm{min}$ ) et couleur des tubes. (3) Grande bobine de mélange, 29 spires, $\varnothing$ ext. $4 \mathrm{~mm}$. (4) Petite bobine de mélange, 12 spires, $\varnothing$ ext. $4 \mathrm{~mm}$. (5) Serpentin de délai simple, $\varnothing$ int $=1,6 \mathrm{~mm}$, température ambiante. (6) Colorimètre, $623 \mathrm{~nm}$. (7) Enregistreur.

* En l'absence de Brij 35 dans l'échantillon, ce réactif peut être supprimé, ainsi que le tuyau noir de la première bobine (4). Dans ce cas la seconde bobine (4) doit être remplacée par une grande bobine de mélange. 
EDTA CuNa 2 au lieu de EDTA Na $\mathrm{Na}_{2}+\mathrm{CuSO}_{4}$ permet de doubler la sensibilité mais, même en faisant varier les conditions opératoires, nous n'avons pu obtenir une courbe étalon linéaire. Pour le dosage en série, prélever $36 \mathrm{~s}$, rincer $90 \mathrm{~s}$. Pour la chromatographie, nous avons utilisé comme standard un tripeptide, le glutamyl-glycyl-phénylalanine et l'avons étalonné par rapport à l'albumine de sérum bovin.

\section{Produits chimiques :}

Sérum albumine bovine Sigma, glutamyl-glycyl-phénylalanine Sigma, acide éthylènediamine tétraacétique disodique (EDTANa ${ }_{2}$ ) p.a. Prolabo, sulfate cuivrique $5 \mathrm{H}_{2} \mathrm{O}$ p.a. Prolabo, sodium hydroxyde p.a. en pastilles Prolabo, sodium carbonate anhydre p.a. Prolabo, réactif de Folin-Ciocalteu Merk, sodium dodécylsulfate Merk, acide benzoïque p.a. Prolabo.

\section{Composition des réactifs :}

- Réactif cuivrique : préparer les solutions $A$ et $B$ suivantes :

A $\left\{\begin{array}{l}\mathrm{EDTANa}_{2}=233 \mathrm{mg} \\ \mathrm{CuSO}_{4} 5 \mathrm{H}_{2} \mathrm{O}=78 \mathrm{mg} \\ \mathrm{H}_{2} \mathrm{O} \text { distillée }=100 \mathrm{ml}\end{array} \quad B\left\{\begin{array}{l}\mathrm{Na}_{2} \mathrm{CO}_{3}=20 \mathrm{~g} \\ \mathrm{NaOH}=4 \mathrm{~g} \\ \mathrm{H}_{2} \mathrm{O} \text { distillée }=600 \mathrm{ml}\end{array}\right.\right.$

Mélanger $\mathrm{A}$ et $\mathrm{B}$, ajuster à $1 \mathrm{I}$ avec de l'eau distillée.

- Réactif de Folin-Ciocalteu : diluer 2 fois avec de l'eau distillée.

- Solution de sodium dodécylsulfate (SDS) : peser avec un masque $100 \mathrm{~g}$ de SDS, ajouter environ $800 \mathrm{ml}$ d'eau distillée, dissoudre à chaud, refroidir, ajuster à 1 I. Ce réactif peut être supprimé en l'absence de Brij 35 dans l'échantillon.

- Albumine de sérum bovin : préparer une gamme étaion de 50 à $100 \mu \mathrm{g} / \mathrm{ml}$ en solution dans l'acide benzoïque 1 p. 1000 pour faciliter la conservation.

- Glutamyl-glycyl-phénylalanine : dissoudre à chaud dans une solution d'acide benzoïque 1 p. 1000 .

- Acide benzoïque : dissoudre à chaud $1 \mathrm{~g}$ d'acide benzoïque pour 11 d'eau distillée.

\section{Références}

ARONSON M., 1952. Transgalactosidation during lactose hydrolysis. Arch. Biochem. Biophys., 39, 370-378.

ATTAIX D., 1979. Evolution de la synthèse protéique in vivo dans les tissus digestifs de l'agneau au cours du développement. Mém. D.E.A., Univ, Clermont-Ferrand II.

BELLA A., Jr., KIM Y. S., 1972. Rat small intestine mucin; isolation and characterization of water-soluble mucin fraction. Arch. Biochem. Biophys., 150, 679-689.

BESLE J. M., 1974. Séparation des oses, des di et triholosides par chromatographie d'échanges d'ions. Ann. Biol. anim. Bioch. Biophys., 14, 545-573.

BESLE J. M., LASSALAS B., THIVEND P., 1980. Digestion des glucides de la levure d'alcanes par le veau préruminant. Reprod. Nutr. Dévelop., 20, 1401-1414.

BESLE J. M., LASSALAS B., THIVEND P., 1981. Digestion des glucides de la féverole par le veau préruminant. Reprod. Nutr. Dévelop., 21, 25-45.

BESLE J. M., PITIOT M., 1976. Extraction et purification des glucides, application à divers aliments dérivés du soja. Ann. Biol. anim. Bioch. Biophys., 16, 753-772.

BESLE J. M., POUGHEON M., BAYLE E., THIVEND P., 1981. Etude des glucides et des glycoprotéines endogènes des digesta chez le veau préruminant. Reprod. Nutr. Dévelop., 21, 859.

COMBe E., PATUREAU-MIRAND P., BAYLE G., PION R., 1980. Influence de l'aliment et de la microflore sur la teneur en sucres aminés des contenus digestifs et des fèces chez le rat, I'agneau et le veau préruminant. Reprod. Nutr. Dévelop., 20, 1707-1715.

CONTREPOIS M., GOUET P., 1973. La microflore du tube digestif du jeune veau préruminant : dénombrement de quelques groupes bactériens à différents niveaux du tube digestif. Ann. Rech. vet., 4, 161-170. 
COOMBE N. B., SMITH R. H., 1973. Absorption of glucose and galactose and digestion and absorption of lactose by the preruminant calf. Br. J. Nutr., 30, 331-345.

DEGAND P., GAVERIAUX M., HAVEZ R., 1972. Définition biochimique des sulfomucines et des sialomucines de l'intestin grêle du porc. C.R. Soc. Biol., 166, 622-627.

FALK K. E., KARLSSON K. A., LEFFLER H., SAMUELSON B. E., 1979. Specific pattern of glycosphingolipids enriched in a mucosa scraping of human intestine. Febs Letters, 101, 273-276.

FOURNET B., FIAT A. M., MONTREUIL J., JOLLES P., 1975. The sugar part of caseins from cow milk and colostrum and its microheterogeneity. Biochimie, 57, 161-167.

GAILLARD B. D. E., VAN WEERDEN E. J., 1976. The digestion of yeast cell wall polysaccharides in veal calves. Br. J. Nutr., 36, 471-478.

HUGUET R., SOLĖRE M., RÉMY-HEINTZ N., 1975. Isolement et composition d'une glycoprotéine de muqueuse gastrique d'agneau. Biochimie, 57, 105-112.

JENNESS R., 1974. The composition of milk, 23-95. In LARSON B. L., SMITH V. R., Lactation, a comprehensive treatise. III. Nutrition and biochemistry of milk, maintenance. Acad. Press, New York London.

JOUANY J. P., THIVEND P., 1972. Evolution post-prandiale de la composition glucidique des corps microbiens du rumen en fonction de la nature des glucides du régime. Il. Les bactéries. Ann. Biol. anim. Bioch. Biophys., 12, 679-683.

KATHAN R. H., ADAMANLY A., 1967. Comparison of human MM, NN and MN blood group antigens. J. biol. Chem., 242, 1716-1722.

LOWRY O., ROSEBROUGH N. J., FARR A. L., RANDALL X. J., 1951. Protein measurement with the folin phenol reagent. $J$. biol. Chem., 193, 265-275.

MONTREUIL J., 1957. Glycoprotéides. Bull. Soc. Chim. biol., 39, suppl. III, 1-92.

MONTREUIL J., KOBUS W., 1960. Variations des constituants glucidiques du lait de vache au cours de la lactation. C.R. Soc. Biol., 154, 2075-2078.

MONTREUIL J., SPIK G., 1968. Microdosage des glucides, monographie $n^{\circ} 2$ : Méthodes chromatographiques et électrophorétiques de dosage des glucides constituant les glycoprotéines. Monogr. Lab. Chimie Biol., Fac. Sc., Lille.

MOON A. W., JOEL D. D., 1975. Epithelial cell migration in the small intestine of sheep and calves. Am. J. vet. Res., 36, 187-189.

PALlAVICINI G., CETTA G., CALATRONI A., SINIGAGLIA F., 1972. Recerche sulia struttura di Glycopeptidi di mucosa gastrica di Suino. Bull. Soc. ital. Biol. sper., 48, 1253-1256.

PARRY G., 1978. Membrane assembly and turnover, 261-326. In ROODYN D. B., Subcellular biochemistry, 5, Plenum Press, New York and London.

PEYRAUD J. L., 1981. Communication personnelle.

PORTER P., NOAKES D. E., 1970. Immunoglobulin IgA in bovine serum and external secretions. Biochim. biophys. Acta, 214, 107-116.

ROBERTS H. R., Mc FARREN F., 1953. The chromatographic observation of oligosaccharides formed during the lactase hydrolysis of lactose. J. dairy Sci., 42, 620-632.

SNARY D., ALLEN A., 1971. Studies on gastric mucoproteins. The isolation and characterization of the mucoprotein of the water-soluble mucus from pig cardiac gastric mucosa. Biochem. J., 123, 845-853.

SNARY D., ALLEN A., 1972. Studies on gastric mucoproteins. The production of radioactive mucoproteins by pig gastric mucosal scrapings in vitro. Bioch. J., 127, 577-587.

SPIRO R. G., 1973. Glycoproteins. Adv. Protein Chem., 27, 349-467.

STRECKER G., MONTREUIL J., 1979. Glycoprotéines et glycoprotéinoses. Biochimie, 61, 1199-1246.

TALMANT A., 1970. Communication personnelle.

TERNOUTH J. H., SIDDONS R. C., TOOTHILL J., 1971. Pancreatic secretion in the milk fed calf. Proc. Nutr. Soc., 30, 89 A-90 A.

WALLENFELS K., 1951. Enzymatische synthese von oiligosaccharides aus dissachariden. Naturwissenschaften, 38, 306-307.

WANG C. S., SMITH R. L., 1975. Lowry determination of protein in the presence of triton $\mathrm{X}-100$. Anal. Biochem., 63, 414-417. 